\section{Pathologies humaines et récepteurs NOTCH}

Cédric Le Caignec

$>$ La voie de signalisation Notch est une cascade de signalisation essentielle au développement de la plupart des organismes multicellulaires $[1,11]$. Chez l'Homme, il existe quatre récepteurs NOTCH (NOTCH1-4) et au moins cinq ligands de NOTCH (JAGl [jagged], JAG2, DLL1 [delta-like], DLL3 et DLL4). Les récepteurs NOTCH sont des protéines transmembranaires comprenant un domaine extracellulaire principalement constitué de multiples motifs EGF-like (epidermal growth factor), une partie transmembranaire unique et un domaine intracellulaire contenant plusieurs motifs ankyrine, des signaux d'adressage nucléaire et un domaine carboxy-terminal proline/glutamic acid/serine/threonine-rich (domaine PEST) (Figure IA). La voie de signalisation NOTCH est activée lors d'un contact entre deux cellules : le ligand enchâssé dans une première cellule se lie au récepteur NOTCH d'une deuxième cellule induisant un clivage du récepteur à proximité de sa partie transmembranaire. La partie intracellulaire est ensuite adressée au noyau et va réguler l'expression de nombreux gènes en association avec d'autres cofacteurs transcriptionnels. Le domaine PEST joue un rôle essentiel dans la stabilité de la partie intracellulaire de NOTCH. La phosphorylation du domaine PEST entraîne sa dégradation en l'orientant vers le protéasome. Des mutations au sein de différents récepteurs NOTCH ont été identifiées dans plusieurs pathologies humaines.

NOTCH2 et syndrome de Hajdu-Cheney Le syndrome de Hajdu-Cheney est une pathologie osseuse rare associant plu- sieurs signes : un visage évocateur, des anomalies radiologiques (une ostéoporose sévère, un défaut d'ossification du crâne et une acro-ostéolyse qui peut se manifester par une disparition progressive des phalanges distales) et une chute prématurée de la dentition définitive [2]. D'autres anomalies sont parfois observées, parmi lesquelles une fente palatine, une malformation cardiaque congénitale, des reins polykystiques, des anomalies génitales, oculaires ou digestives. Une cinquantaine de patients ont été rapportés dans le monde dont la plupart sont atteints des formes sporadiques de la maladie. D'exceptionnelles formes familiales ont également été observées suggérant une transmission autosomique dominante. Dans un travail récent, nous avons analysé les exomes de six patients de six familles non apparentées par technique de séquençage haut débit [3]. L'analyse des données nous a conduits à identifier des mutations hétérozygotes au sein du gène NOTCH2 chez 5 des 6 patients. Nous avons pu montrer que la mutation est apparue de novo chez deux patients dont les parents étaient normaux. Dans les formes familiales, les patients présentent tous la mutation identifiée chez le cas index tandis qu'elle est absente chez les personnes saines. Ces résultats ainsi que ceux, très similaires, d'une équipe concurrente indiquent que le syndrome de Hajdu-Cheney est lié à des mutations de NOTCH2 [4]. Au-delà du rôle de ce gène dans ce syndrome rare, ces résultats établissent un lien entre NOTCH2 - et plus généralement la voie Notch et l'ostéoporose commune.
CHU de Nantes, Service de génétique médicale, 9, quai Moncousu, 44093 Nantes, France. Inserm, UMR915, institut du thorax, Nantes ; Université de Nantes; CNRS, ERL3147, Nantes, F44007, France.

cedric.lecaignec@univ-nantes.fr

De façon intéressante, toutes les mutations identifiées créent un codon stop prématuré (mutations non-sens ou entraînant un décalage du cadre de lecture) localisé dans le dernier exon du gène et sont responsables d'une protéine tronquée (Figure 1B). Le fait que ces mutations soient situées dans le dernier exon du gène explique que l'ARN messager (ARNm), bien qu'anormal, ne soit pas reconnu par le système de dégradation des ARNm non-sens (NMD, nonsense mediated decay). Une des fonctions du système NMD est de pouvoir identifier la présence d'un codon stop prématuré situé avant le dernier exon d'un ARNm anormal et ainsi de permettre sa dégradation $[5,12]$. En revanche, lorsque le codon stop prématuré est situé dans le dernier exon d'un gène, il échappe à ce système de reconnaissance. La protéine tronquée qui est traduite peut avoir perdu un domaine fonctionnel important mais conservé d'autres domaines fonctionnels localisés en amont du codon stop prématuré. Dans le cas du syndrome de Hajdu-Cheney, la partie intracellulaire de NOTCH2, née du clivage de la protéine, conserve les motifs ankyrine et donc certaines fonctions de régulation transcriptionnelle. En revanche, elle est amputée du domaine PEST, ce qui limite sa dégradation. Bien que cela reste à démontrer, il est donc très probable que les mutations observées chez les patients atteints du syndrome de Hajdu-Cheney soient des mutations activatrices.

\section{NOTCH3 et syndrome de CADASIL}

Le syndrome de CADASIL (cerebral autosomal dominant arteriopathy with sub- 


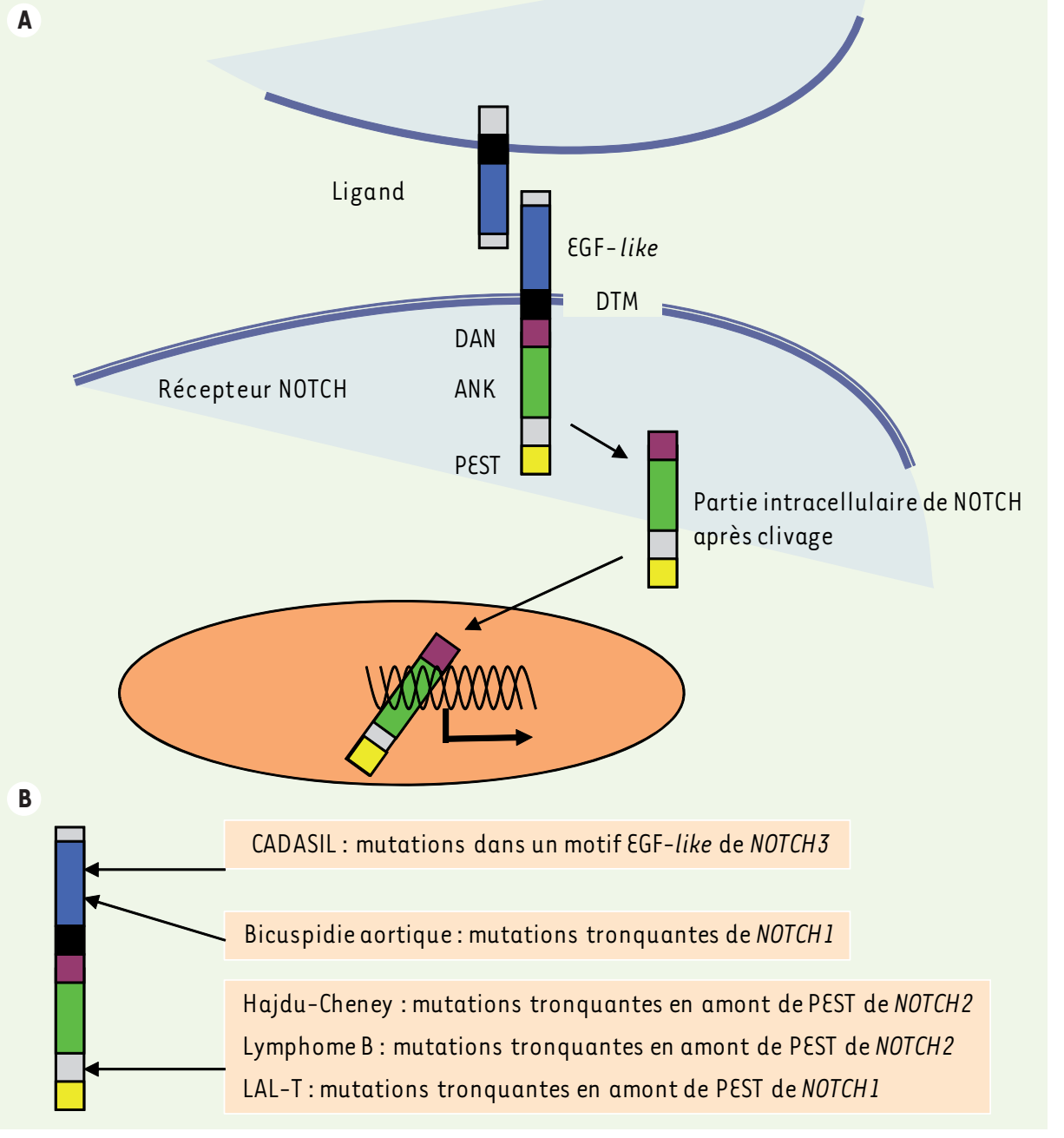

Figure 1. A. Mécanisme d'action des récepteurs NOTCH. La fixation du ligand au récepteur NOTCH entraîne son clivage en amont du domaine transmembranaire. La partie intracellulaire clivée migre dans le noyau de la cellule où elle régule l'activité transcriptionnelle de nombreux gènes. EGF-like : epidermal growth factor like; DTM : domaine transmembranaire; DAN : domaine d'adressage nucléaire ; ANK : motif ankyrine; PEST : domaine PEST. $\boldsymbol{B}$. Localisation des mutations des récepteurs NOTCH en pathologie humaine.

patients porteurs de la mutation présentent une autre forme de malformation cardiaque congénitale telle qu'une communication interventriculaire, une tétralogie de Fallot, une hypoplasie du ventricule gauche ou une sténose valvulaire mitrale. Des études fonctionnelles complémentaires suggèrent un mécanisme perte de fonction de ces mutations.

cortical infarcts and leukoencephalopathy) est une maladie génétique rare de transmission autosomique dominante. Elle associe des migraines à des accidents vasculaires cérébraux, essentiellement de type ischémique et conduisant à des lésions de la substance blanche du cerveau. L'évolution est marquée par une récidive des symptômes vasculaires, une possibilité d'atteinte cognitive et une atteinte psychiatrique (syndrome dépressif, parfois accès maniaques ou mélancoliques). Le diagnostic, suspecté sur les données cliniques, est confirmé par l'IRM (imagerie par résonance magnétique) qui met en évidence des infarctus de type lacunaire. Une mutation hétérozygote au sein du gène NOTCH3 est identifiée chez la majorité des patients atteints du syndrome de
CADASIL [6]. Les mutations conduisent à une perte ou à un gain d'une cystéine dans un des motifs EGF-like du domaine extracellulaire de NOTCH3 (Figure IB). Ce type de mutation, associé au fait qu'il n'a pas été observé de délétions du gène chez les patients, suggère que les mutations sont des mutations gain de fonction plutôt que perte de fonction.

\section{NOTCH1 et pathologie}

valvulaire aortique

L'étude de deux grandes familles au sein desquelles plusieurs patients présentaient une bicuspidie aortique ayant parfois évolué vers un rétrécissement aortique calcifié a conduit à l'identification de mutations non-sens de NOTCHI [7] (Figure 1B). En dehors de l'anomalie valvulaire aortique, certains

\section{NOTCH et hémopathies malignes}

Environ la moitié des leucémies aiguës lymphoblastiques T (LAL-T) sont associées à des mutations hétérozygotes de NOTCHI [8] tandis qu'environ $8 \%$ des patients atteints d'un lymphome $B$ à grandes cellules présentent une mutation hétérozygote de NOTCH2 [9]. De la même façon que dans le syndrome de Hajdu-Cheney, la majorité des mutations conduisent à une protéine tronquée ayant perdu le domaine PEST (Figure 1B). Des études fonctionnelles ont démontré que ces mutations étaient activatrices. Ces découvertes moléculaires ont conduit à tester in vivo un inhibiteur de gamma-sécrétase dans un modèle de xénogreffe chez la souris SCID (severe combined immunodeficiency). Cet inhibiteur supprime le clivage de 
Notchl et donc le transfert au noyau de l'hétérodimère membranaire Notch. L'effet oncogénique de Notchl, lié à son activation constitutive, est ainsi empêché. Après inoculation à la souris par voie sous-cutanée de diverses lignées de LAL-T humaines, l'administration per os pendant au moins 30 jours de cet inhibiteur induit la régression totale ou partielle des tumeurs [10].

\section{Conclusions}

Les récepteurs de la voie Notch jouent un rôle majeur dans la différenciation cellulaire, notamment chez les vertébrés. L'identification de mutations au sein de NOTCH1, 2 et 3 a permis d'expliquer plusieurs pathologies humaines constitutionnelles ou acquises. Pour certaines d'entre elles, les mutations sont activatrices, ce qui permet d'envisager des solutions thérapeutiques grâce à l'utilisation d'inhibiteurs de gamma-sécrétase. $\diamond$

Human diseases and NOTCH receptors
CONFLIT D'INTÉRÊTS

L'auteur déclare n'avoir aucun conflit d'intérêts concernant les données publiées dans cet article.

\section{RÉFÉRENCES}

1. Kopan R, llagan MX. The canonical Notch signaling pathway: unfolding the activation mechanism. Cell $2009 ; 137: 216-33$

2. Brennan AM, Pauli RM. Hajdu-Cheney syndrome: evolution of phenotype and clinical problems. Am J Med Genet $2001 ; 100: 292-310$.

3. Isidor B, Lindenbaum P, Pichon 0 , et al. Truncating mutations in the last exon of $\mathrm{NOTCH} 2$ cause a rare skeletal disorder with osteoporosis. Nat Genet 2011 $43: 306-8$

4. Simpson MA, Irving MD, Asilmaz $\varepsilon$, et al. Mutations in NOTCH2 cause Hajdu-Cheney syndrome, a disorder of severe and progressive bone loss. Nat Genet 2011; $43: 303-5$.

5. Holbrook JA, Neu-Yilik G, Hentze MW, et al. Nonsensemediated decay approaches the clinic. Nat Genet $2004 ; 36: 801-8$

6. Joutel A, Corpechot C, Ducros A, et al. Notch3 mutations in CADASIL, a hereditary adult-onset condition causing stroke and dementia. Nature 1996 ; $383: 707-10$.

7. Garg V, Muth AN, Ransom JF, et al. Mutations in Notch l cause aortic valve disease. Nature $2005 ; 437$ : 270-4.

8. Weng AP, Ferrando AA, Lee $W$, et al. Activating mutations of NOTCHl in human T cell acute lymphoblastic leukemia. Science 2004 ; 306 : 269-71.
9. Lee SY, Kumano K, Nakazaki K, et al. Gain-of-function mutations and copy number increases of Notch2 in diffuse large B-cell lymphoma. Cancer Sci 2009; 100 : 920-6.

10. Masuda S, Kumano K, Suzuki T, et al. Dual antitumor mechanisms of Notch signaling inhibitor in a T-cell acute lymphoblastic leukemia xenograft model. Cancer Sci 2009 ; 100 : 2444-50.

11. Mayeuf A, Relaix F. La voie Notch : du développement à la régénération du muscle du squelette. Med Sci (Paris) $2011 ; 27: 521-6$.

12. Camier $S$, Séraphin B. Détruisez ce message (ARN) après l'avoir lu! Med Sci (Paris) $2007 ; 23: 850-6$

\section{Apprenez à respirer à vos enfants}

\section{Jean-Paul Allaux}

Les maladies ORL avec leur prolongement au niveau pulmonaire sont malheureusement de plus en plus répandues de nos jours et à tout âge.

La pollution atmosphérique, les appartements surchauffés ou enfumés par des parents tabagiques, les rues des villes saturées de gaz d'échappement... tous ces maux contemporains bien connus sont bien souvent responsables de l'installation de ces affections dès la petite enfance. Ils freinent par ailleurs le développement harmonieux du corps e $n$ installant parfois des déformations vertébrales mais aussi du palais et des mâchoires provoquant une mauvaise implantation des dents.

Fruit d'une longue expérience, ce livre se veut avant tout pratique. De nombreux exercices spécifiques à faire à la maison ou à l'école, seul ou en groupe, permettront aux éducateurs, parents et enseignants de trouver remède à ces différentes affections et d'aider efficacement les enfants à s'épanouir pleinement.

Un livre pour apprendre à respirer à nos enfants.

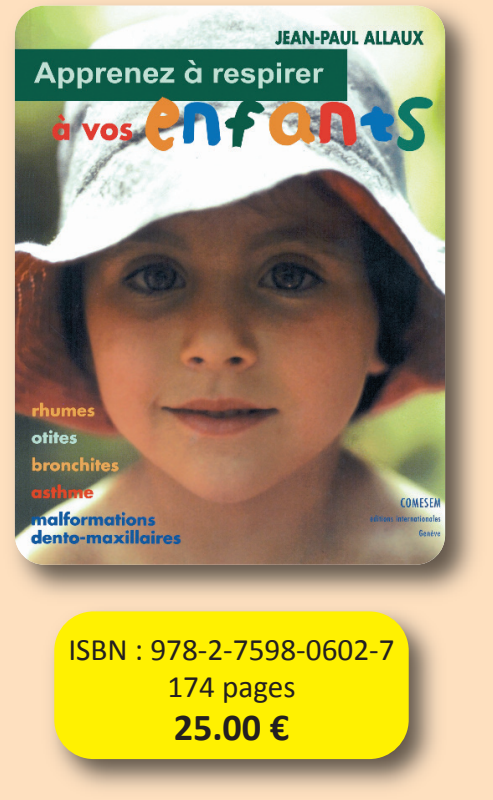

\section{Commandez directement sur : www.edition-sciences.com}

